\title{
Phlegmasia dolens
}

GC Ukala ${ }^{1}$, H Ryan Gregory ${ }^{2}$

Consent: Written consent obtained from patient.

Correspondence to:

GC Ukala

Declaration of interests: No conflict of interests declared

Mandeville Regional

Hospital

Mandeville

Jamaica

Email:

ukalasangel@hotmail.com

A 30-year-old female presented with swelling and pain to the left lower limb. She had no history of recent long travel, use of oral contraceptives, smoking or trauma. Venous Doppler ultrasound confirmed extensive left lower limb deep venous thrombosis. Initial thrombophilia screen was negative. She was anticoagulated with heparin and warfarin achieving international normalized ratio of 2.21 .

One week later, still fully anticoagulated, she re-presented with worsening pain and swelling to the same lower limb with engorged superficial veins in the groin (Figure 1).

A diagnosis of phlegmasia cerulea dolens (extreme lowerextremity deep vein thrombosis capable of causing critical limb ischemia/limb loss ${ }^{1}$ ) was considered. Subsequent abdomino-pelvic CT showed compression of the left iliac vein by the right iliac artery (May-Thurner syndrome ${ }^{2}$ ) (Figure 2).

Endovascular catheter-directed thrombolysis with alteplase was given followed by low dose heparin and stenting at point of compression by vascular surgeons with excellent results (Figure 3).

A high index of suspicion and a comprehensive diagnostic approach are critical for detecting May-Thurner syndrome in phlegmasia. (1)

\section{Acknowledgements}

Thanks to Dr Sundeep Shah for Figure 2.
Figures 1-3 showing the progression and resolution of the patient's condition
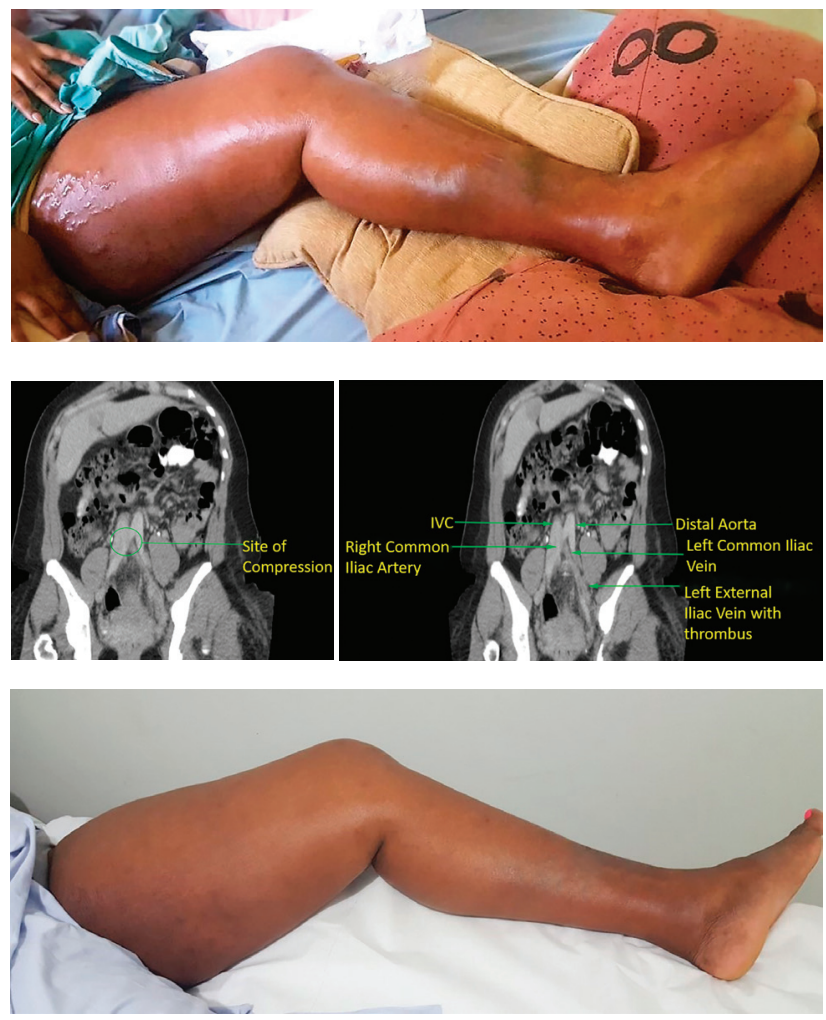

\section{References}

1 Chaar ClO. Phlegmasia Alba and Cerulea Dolens. Updated December 2016. http://emedicine.medscape.com/article/461809-overview

2 Peters MD, Syed KR, Katz M et al. May-Thurner syndrome: a not so uncommon cause of a common condition. Proc (Bayl Univ Med Cent) 2012: 25: 231-3. 